\title{
Sequestering in models of F-term uplifting*
}

\author{
Hiroyuki Abe ${ }^{\dagger}$ \\ Yukawa Institute for Theoretical Physics, Kyoto University, Kyoto 606-8502, Japan
}

\begin{abstract}
We discuss the nature of sequestering supersymmetry breaking sectors in a certain class of moduli stabilization in supergravity/string models, where a negative vacuum energy of the nonperturbative moduli potential is canceled by dynamically generated F-terms. Two illustrating examples are shown to sketch the issues around the supersymmetry breaking, flavors and sequestering within such a framework.
\end{abstract}

${ }^{*}$ Talks given at PASCOS08 (the 14th International Symposium on Particles, Strings and Cosmology, Perimeter Institute, Waterloo, Canada, June 2-6, 2008) and SUSY08 (the 16th International Conference on Supersymmetry and the Unification of Fundamental Interactions, the COEX Center, Seoul, Korea, June 16-21, 2008).

†E-mail address: abe@yukawa.kyoto-u.ac.jp 


\section{Introduction}

The low-scale supersymmetry (SUSY) is one of the best solutions to the weak-Planck hierarchy problem, and then a good candidate for the physics at the $\mathrm{TeV}$ scale beyond the standard model (SM). The minimal SUSY SM (MSSM) unifies three gauge coupling constants at a certain high energy scale, and provides a dark matter candidate as the lightest SUSY particle. The existence of SUSY is also predicted by the only known consistent framework of quantum gravity, i.e., superstring theory.

In constructing realistic SUSY models, there are several conditions to be satisfied. First of all, in order to avoid the so-called supertrace theorem, the SUSY would be broken in the hidden sector and communicated to the visible (MSSM) sector by some messenger fields. The low-energy SUSY particle spectrum is determined by the mediation mechanism. One of the severest constraints on the mediation structure comes from the observations of flavor changing processes, i.e., SUSY flavor violations.

The hidden sector should not couple to the visible sector, by definition, through tree-level renormalizable interactions. Such a structure would be naturally realized in a higher-dimensional spacetime, where both sectors are somehow separated from each other geometrically in extra dimensions [1], which is sometimes referred to as sequestering. The higher-dimensional spacetime is also predicted by string theory. Aside from the anomaly mediation [1, 2], the most natural candidate for the messenger in such a situation is the moduli which govern the size and the shape of the extra dimensions. A more model-dependent candidate is SM-charged massive fields if they exist, i.e., the gauge mediation.

Because moduli originate from the higher-dimensional gravity multiplet, the dominant interactions with the visible sector as well as with the hidden sector appear as tree-level nonrenormalizable terms, which are determined by the geometry of the extra dimensions. The moduli, as it stands, are flat-directions of the tree-level or the perturbative potential, which should be stabilized by some mechanisms such as nonperturbative dynamics. The magnitude of the SUSY breaking mediated by moduli is also determined by the stabilization mechanism. One of the most challenging issues around the moduli stabilization is a realization of an almost vanishing vacuum energy at a minimum of the moduli potential, required by the observation of our universe.

\section{$2 \quad$ Uplifting}

Most of $N=1$ compactifications of supergravity/string have moduli in the low-energy effective theory. Many of them can be stabilized by turning on some fluxes/torsions in extra-dimensional space, while some of them can not. The remaining moduli would be stabilized by field theoretical nonperturbative effects such as gaugino condensations. However, it is known that such effects generically yield negative vacuum energies at the minima of moduli potential, none of which corresponds to our universe.

There has been proposed a systematic way for fixing the moduli at a supersymmetry breaking minimum with a vanishing vacuum energy [3], decoupling the SUSY breaking sector from the moduli as well as from the MSSM sector. In this case, the SUSY breaking 
(hidden) sector is also called an uplifting sector, because the SUSY breaking energy lifts the negative energy of the nonperturbative moduli potential to be vanishing. A simple and natural candidate for the source of the uplifting energy is the F-terms of dynamical SUSY breaking sectors. We call this scenario the F-term uplifting. It is notable that there would exist a large number of (metastable) SUSY breaking states in supergravity/string models caused by, e.g., SUSY gauge dynamics [4], that can provide the uplifting F-terms in a controllable manner ( see, e.g., Ref [5, 6]).

\section{Low energy effective theory}

The relevant quantities to our discussion in a generic four-dimensional (4D) $N=1$ effective supergravity are

$$
\begin{aligned}
\Omega= & \Omega_{0}(T, \bar{T})+Y_{X \bar{X}}(T, \bar{T})|X|^{2}+Y_{I \bar{J}}\left(T, \bar{T},|X|^{2}\right) Q^{I} Q^{\bar{J}} \\
& \quad+L_{I J}\left(T, \bar{T},|X|^{2}\right) Q^{I} Q^{J}+\text { h.c. }+\cdots, \\
W= & W_{0}(T)+f(T) X+\mu_{I J}(T) Q^{I} Q^{J} / 2 !+\lambda_{I J K}(T) Q^{I} Q^{J} Q^{K} / 3 !+\cdots, \\
f_{a}= & l_{a}+k_{a} T+\cdots,
\end{aligned}
$$

where $K=-3 \ln (-\Omega / 3)$ is the Kähler potential, $W$ is the superpotential and $f_{a}$ are the gauge kinetic functions determining the gauge couplings associated with vector multiplets labelled by the index $a$ as $\left\langle\operatorname{Re} f_{a}\right\rangle=1 / g_{a}^{2}$. The chiral multiplets $T, X$ and $Q^{I}$ represent the light moduli, the uplifting (SUSY breaking) and the visible (MSSM) sectors, respectively. The ellipses denote irrelevant or higher-order terms in powers of $X$ and $Q^{I}$, some of which might be suppressed due to some (approximate) symmetries, such as an $U(1)_{R^{-}}$symmetry which is responsible for the dynamical SUSY breaking and assumed to be broken by $W_{0}$ [7].

From these functions, we can evaluate soft SUSY breaking terms like the gaugino masses $M_{a}$, the scalar masses $m_{I}^{2}$, and the scalar trilinear couplings $A_{I J K}$ (A-terms) in the MSSM sector as

$$
\begin{aligned}
M_{a} & =F^{i} \partial_{i} \ln \left(\operatorname{Re} f_{a}\right), \\
m_{I}^{2} & =-F^{i} \bar{F}^{\bar{j}} \partial_{i} \partial_{\bar{j}} \ln Y_{I \bar{I}}, \\
A_{I J K} & =F^{i} \partial_{i} \ln \left(\lambda_{I J K} / Y_{I \bar{I}} Y_{J \bar{J}} Y_{K \bar{K}}\right),
\end{aligned}
$$

where the indices $i, j$ label the fields with nonvanishing F-terms such as the uplifting field $X$ and the moduli $T$. Here we set $Y_{I \bar{J}}=0$ for $J \neq I$ to reduce the expressions.

\subsection{IIB orientifold model}

A concrete uplifting scheme was first proposed [3] in the framework of warped flux compactifications [8]. In the type IIB orientifold model, the shape moduli as well as the dilaton can be fixed with heavy masses by introducing three-form flux in extra dimensions (Calabi-Yau three-folds). On top of that, if we consider gaugino condensations on D-branes wrapping four-cycles, the corresponding size moduli $T$ would receive a superpotential $W_{\mathrm{np}}(T)=\sum_{n}^{n_{\mathrm{np}}} A_{n} e^{-a_{n} f_{a}(T)}$ where $A_{n} \sim \mathcal{O}(1)$ and $a_{n} \sim \mathcal{O}\left(4 \pi^{2}\right)$. Combining this 
with possible constant contributions induced by the three-form flux or nonperturbative effects on three-branes, the effective moduli superpotential is given by $W_{0}=c+W_{\mathrm{np}}(T)$. Assuming $K_{0}(T), \partial_{T} K_{0}(T), \partial_{T} \partial_{\bar{T}} K_{0}(T), \ldots=\mathcal{O}(1)$ for $T=\mathcal{O}(1)$, the effective $N=1$ moduli potential has only SUSY preserving minima with (semi-)negative vacuum energies without any fine tuning as mentioned above.

In order to lift one of the minima to have a vanishing vacuum energy and break SUSY, we put an anti D3-brane at the tip of a warped throat induced by the three-form flux. The anti-brane is sequestered from the size moduli $T$ as well as from the MSSM sector due to the warped geometry, if we assume, e.g., that the MSSM sector is put far from the warped throat in the extra dimensions. In this case, the effect of the antibrane appears just as an uplifting energy which breaks $N=1$ SUSY explicitly in the 4D effective theory, and soft terms are determined by light moduli F-terms estimated as, e.g., $F^{T} \approx m_{3 / 2} / \ln \left(M_{P l} / m_{3 / 2}\right)$ for $n_{\mathrm{np}}=1$ [9] (see Ref. [10] for $l_{a} \neq 0$ ). If we assume that only the heavy moduli (e.g., shape moduli in this case) distinguishes the SM flavors, there would be no dangerous SUSY flavor violations [11]. However, note before mentioning the SUSY flavor violations that, it is still challenging to obtain even the SM flavor structure such as proper generation numbers and the realistic Yukawa coupling matrices within the framework of IIB orientifold models.

Instead of the anti-brane, we can think of the F-term uplifting. We consider the situation that a dynamical SUSY breaking occurs somewhere in extra dimensions, which might be represented by $X$ at a low energy with the nonvanishing Polonyi term $f \neq 0$ in the effective superpotential. We can tune the parameters $c$ and $f$ so that the F-term of $X$ cancels the negative energy of the moduli potential $W_{0}$. However in this case, direct couplings between $X$ and $Q^{I}$ through $Y_{I \bar{J}}\left(T, \bar{T},|X|^{2}\right)$ could appear in general, depending on the explicit construction of $X$ and $Q^{I}$ sectors. Then, the issues of sequestering is model dependent and the derivation of $Y_{I \bar{J}}$ model by model might be quite complicated with six extra dimensions.

\section{$3.25 \mathrm{D}$ orbifold model}

The five-dimensional (5D) supergravity provides the simplest illustrating framework to study the issues of dynamical uplifting and sequestering, because of the singleness of the extra dimension. Moreover, it is known that a certain class of realistic Yukawa matrices can be realized due to the wavefunction localization caused by gauging MSSM matter fields under the graviphoton.

Thanks to the off-shell dimensional reduction [12] which is based on the $N=1$ superspace [13], we can derive $K, W$ and $f_{a}$ in a systematic way for an arbitrary setup of the 5D supergravity on orbifold $S^{1} / Z_{2}$. When both $X$ and $Q^{I}$ originate from 5D hypermultiplets charged under the graviphoton, we find $\Omega_{0}=-3(T+\bar{T}) / 2, Y_{X \bar{X}}=\left(1-\left|e^{-c_{X} T}\right|^{2}\right) / c_{X}$, $L_{I J}=0$ and

$$
Y_{I \bar{J}}=\left(\frac{1-\left|e^{-c_{I} T}\right|^{2}}{c_{I}}+\frac{1-\left|e^{-\left(c_{I}+c_{X}\right) T}\right|}{3\left(c_{I}+c_{X}\right)}|X|^{2}\right) \delta_{I J},
$$

where $c_{X}$ and $c_{I}$ are the graviphoton charges of $X$ and $Q^{I}$ respectively. The parameters in the superpotential such as the Polonyi term $f$ and holomorphic Yukawa couplings $\lambda_{I J K}$ 
appear as constant parameters originating from the superpotential at the fixed points of orbifold. The moduli potential $W_{0}(T)$ would be given in the exactly same form as the above IIB model by assuming bulk (zero mode) and boundary gaugino condensations. Then the argument of the moduli stabilization and uplifting is equivalent to the above IIB case.

An advantage is that we now have exact forms of $Y_{X \bar{X}}$ and $Y_{I \bar{J}}$ which are necessary to calculate $m_{I}^{2}$ and $A_{I J K}$. As mentioned above, we obtain realistic values of physical Yukawa couplings $y_{I J K}=\lambda_{I J K} / \sqrt{Y_{I \bar{I}} Y_{J \bar{J}} Y_{K \bar{K}}}$ with a certain choice of $c_{I}$ due to the wavefunction localizations (see, e.g., Ref. [14] and references therein). By using the above soft term formula, we easily find that the contribution from $X$ to $m_{I}^{2}$ is always tachyonic, and $m_{I}^{2}$ and $A_{I J K}$ vanish at the tree-level in a large $\left|c_{I}\right|$ (or $\left|c_{X}\right|$ ) limit with $c_{I} c_{X}<0$ [15]. The limit corresponds to the situation that $Q^{I}(X)$ localizes more severely toward the opposite fixed point to $X\left(Q^{I}\right)$, and the sequestering is achieved. (In the limit $\left|c_{I}\right|,\left|c_{X}\right| \rightarrow \infty$, the fields $Q^{I}, X$, respectively, become strictly localized at the fixed point.) However, we can also show that the sequestering is incompatible with the wavefunction profile that yields realistic Yukawa matrices. For the realistic Yukawa couplings, the heavy and the light generations need to localize against each other, and then either generation is forced to localize toward the SUSY breaking field $X$, yielding a large tachyonic $m_{I}^{2}$ due to the direct coupling. This might be simply because we have only single extra dimension.

\section{Summary}

The nonperturbative moduli stabilization with uplifting provides a systematic way for realizing a SUSY breaking minimum with a vanishing vacuum energy. We have discussed the feature of sequestering within such a framework based on two illustrating situations. One is a ten-dimensional model that would correspond to a certain low energy limit of IIB superstring (a top-down approach), and another is the 5D orbifold model where all the functions in the low energy effective theory are calculable (a bottom-up approach).

In the former, the realization of SM flavor structure is still challenging issue, while in the latter, the compatibility between the realistic Yukawa structure and sequestering is a nontrivial problem. For IIB models, further studies on flavors based on, e.g., magnetized extra dimensions [16] would be interesting. For 5D models, an extension to the case with multi moduli would solve the problem [17]. Finally, it is important to study these issues also in IIA/heterotic string models or to extend the 5D model to the case with more extra dimensions step by step.

\section{Acknowledgements}

The author would like to thank Tetsutaro Higaki, Tatsuo Kobayashi, Yuji Omura and Yutaka Sakamura for fruitful collaborations. The author is supported by the Japan Society for the Promotion of Science for Young Scientists (No.182496). 


\section{References}

[1] L. Randall and R. Sundrum, Nucl. Phys. B 557, 79 (1999) [hep-th/9810155].

[2] G. F. Giudice, M. A. Luty, H. Murayama and R. Rattazzi, JHEP 9812, 027 (1998) hep-ph/9810442.

[3] S. Kachru, R. Kallosh, A. Linde and S. P. Trivedi, Phys. Rev. D 68, 046005 (2003) [hep-th/0301240].

[4] K. Intriligator, N. Seiberg and D. Shih, JHEP 0604, 021 (2006) [hep-th/0602239].

[5] E. Dudas, C. Papineau and S. Pokorski, JHEP 0702, 028 (2007) hep-th/0610297]

[6] H. Abe, T. Higaki, T. Kobayashi and Y. Omura, Phys. Rev. D 75, 025019 (2007) [hep-th/0611024]; H. Abe, T. Higaki and T. Kobayashi, Phys. Rev. D 76, 105003 (2007) [arXiv:0707.2671 [hep-th]].

[7] K. Intriligator, N. Seiberg and D. Shih, JHEP 0707, 017 (2007) hep-th/0703281];

H. Abe, T. Kobayashi and Y. Omura, JHEP 0711, 044 (2007) arXiv:0708.3148 [hep-th]].

[8] S. B. Giddings, S. Kachru and J. Polchinski, Phys. Rev. D 66, 106006 (2002) hep-th/0105097.

[9] K. Choi, A. Falkowski, H. P. Nilles, M. Olechowski and S. Pokorski, JHEP 0411, 076 (2004) hep-th/0411066]; K. Choi, A. Falkowski, H. P. Nilles and M. Olechowski, Nucl. Phys. B 718, 113 (2005) hep-th/0503216.

[10] H. Abe, T. Higaki and T. Kobayashi, Phys. Rev. D 73, 046005 (2006) [hep-th/0511160].

[11] K. Choi, K. S. Jeong and K. I. Okumura, JHEP 0807, 047 (2008) arXiv:0804.4283 [hep-ph]].

[12] H. Abe and Y. Sakamura, Phys. Rev. D 75, 025018 (2007) hep-th/0610234.

[13] F. Paccetti Correia, M. G. Schmidt and Z. Tavartkiladze, Nucl. Phys. B 709, 141 (2005) hep-th/0408138]; H. Abe and Y. Sakamura, JHEP 0410, 013 (2004) hep-th/0408224]; Phys. Rev. D 71, 105010 (2005) [hep-th/0501183]; Phys. Rev. D 73, 125013 (2006) [hep-th/0511208].

[14] H. Abe, K. Choi, K. S. Jeong and K. i. Okumura, JHEP 0409, 015 (2004) hep-ph/0407005.

[15] H. Abe, T. Higaki, T. Kobayashi and Y. Omura, JHEP 0804, 072 (2008) arXiv:0801.0998 [hep-th]].

[16] D. Cremades, L. E. Ibanez and F. Marchesano, JHEP 0405, 079 (2004) [hep-th/0404229]; H. Abe, T. Kobayashi and H. Ohki, arXiv:0806.4748 [hep-th].

[17] H. Abe and Y. Sakamura, arXiv:0807.3725 [hep-th]. 